\title{
FINDING THE CONVEX HULL OF A SIMPLE POLYGON IN LINEAR TIME*
}

\author{
S. Y. SHIN ${ }^{\dagger}$ and T. C. Woo \\ Department of Industrial and Operations Engineering, The University of Michigan, Ann Arbor. MI 48109. \\ U.S.A.
}

(Received 8 February 1985; in retised form 24 June 1985)

\begin{abstract}
Though linear algorithms for finding the convex hull of a simply-connected polygon have been reported. not all are short and correct. A compact version based on Sklansky 's original idea " and Bykat"s counter-example ${ }^{(8)}$ is given. Its complexity and correctness are also shown.

Convex hull Linear algorithm Computational geometry
\end{abstract}

\section{INTRODUCTION}

There have been many reports on a linear algorithm for finding the convex hull of a simple polygon. Certain versions were prone to counter-examples. In particular, a recent version by Ghosh and Shyamasundar ${ }^{(1)}$ turned out to be incorrect. ${ }^{(2.3)}$ Ideally, an algorithm should be not only correct but also easy to implement. McCallum and Avis, ${ }^{\left({ }^{(1)}\right.}$ for example, reported a version using two stacks. Lee ${ }^{(5)}$ used one stack but the algorithm itself was two pages long. Recently, Graham and $\mathrm{Yao}^{(6)}$ reported a compact algorithm that is said to be similar in spirit to Lee's version. Both Refs $(5)$ and (6) included two types of pocket test. In this paper, we present a version employing only one pocket test.

Perhaps. the simplest version is still the one presented by Sklansky ${ }^{\left({ }^{7}\right)}$ in 1972. After a counter-example by Bykat, ${ }^{(8)}$ sufficiency condition was established by Toussaint and $\mathrm{Avis}^{(9)}$ in 1982 and by Orlowsky ${ }^{(10)}$ in 1983. Almost concurrently, Sklansky gave a modified version $^{\prime 11)}$ but it was later shown to be incorrect by Toussaint and El Gindy. ${ }^{(12)}$ Our search for a simple, concise and correct linear convex hull algorithm traces the following path. For simplicity, we adopt the ideas from the original version by Sklansky. ${ }^{(7)}$ For conciseness, we follow the form of CH-POL by Toussaint and A vis. ${ }^{(9)}$ For correctness, we use the notion of a pocket (or lobe) as in Graham and $\mathrm{Yao}^{(6)}$ (or Lee ${ }^{(5)}$ ) with Bykat's counter-example ${ }^{(8)}$ in mind.

\section{PRELIMINARIES}

Let $P$ be a simple polygon with $n$ vertices. Each vertex $V_{i}, i=0,1,2, \ldots,(n-1)$, is represented by its $X$ and $Y$ coordinates. $\left(X_{i}, Y_{i}\right)$. Let $V_{0}$ be the vertex with

* This work was supported in part by AFOSR under contract F4920-82-C-0089 and in part by IBM Data Systems Division.

t To whom correspondence should be addressed. the minimum $Y$ coordinate. If two or more vertices are tied then we choose among them the vertex with the minimum $X$ coordinate as $V_{0}$. Starting from $V_{0}$ and traversing the boundary $B(P)$ of $P$ in the clockwise order, we label the $j$ th vertex from $V_{0}$ as $V_{i}$, where $i$ is $j$ modulo $n$. These vertices in sequence are maintained as a circular doubly linked list. Throughout this paper we assume the following:

(1) The boundary $B(P)$ of a simple polygon $P$ is traversed in the clock wise order from $V_{0}$.

(2) No three consecutive vertices are colinear.

Definition 2.1. $L\left(P_{i}, P_{j}\right)$ denotes a directed line segment joining two points $P_{i}$ and $P_{i}$ in the direction from $P_{i}$ to $P_{j}$.

Definition 2.2. An edge $E\left(V_{i}, V_{i+1}\right)$ of $P$ is a directed line segment $L\left(V_{i}, V_{i+1}\right)$ joining two adjacent vertices $V_{i}$ and $V_{i+1}$ on $B(P)$. A chain $C\left(V_{i}, V_{j}\right)$ is a sequence of edges $E\left(V_{i}, V_{i+1}\right), E\left(V_{i+1}, V_{i+2}\right), \ldots, E\left(V_{j-1}, V_{j}\right)$ on $B(P)$ in the clock wise order.

Definition 2.3. A vertex $V_{i}$ of $P$ is extreme if $V_{i}$ cannot be expressed as a convex combination of other vertices in $P$, i.e. $V_{i}$ is extreme if and only if

$$
V_{i} \neq \sum_{i \neq 1} \alpha_{j} V_{j} \cdot \sum_{i \neq 1} \alpha_{j}=1, \quad \text { and } \alpha_{j} \geq 0 .
$$

Definition 2.4. The convex hull $C H(P)$ of $P$ is the smallest convex polygon containing $P$.

Definition 2.4 necessarily implies that every vertex of $C H(P)$ is an extreme vertex of $P$. Hence, one way to find $C H(P)$ is to discard all non-extreme vertices. To characterize a non-extreme vertex, we employ the notion of a pocket.

Definition 2.5. A pocket $P K T\left(V_{i}, V_{j}\right)$ is one or more regions bounded by $L\left(V_{i}, V_{j}\right)$ and $C\left(V_{i}, V_{j}\right)$ such that all points in $C\left(V_{i}, V_{j}\right)$ are on or to the right of $L\left(V_{i}, V_{j}\right)$.

We state an interesting property of a pocket due to Graham and Yao. ${ }^{(6)}$

Lemma 2.1. Let $V_{r}$ be in a $P K T\left(V_{i}, V_{j}\right)$. If $V_{r}$ is neither $V_{i}$ nor $V_{j}$, then $v_{r}$ is not an extreme vertex of $P$. 


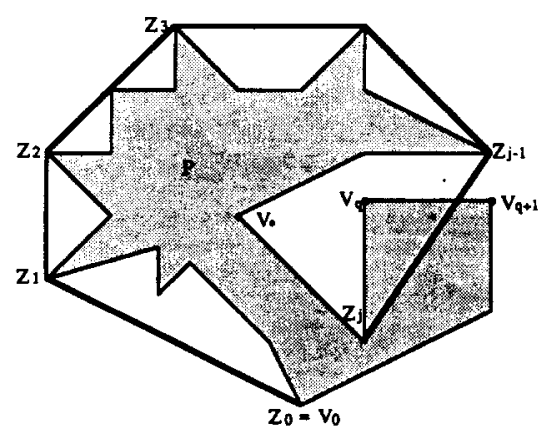

Fig. 1. Vertices of a polygon $P$ and $Z P R\left(V_{0}, V_{q}\right)$.

\section{PROPERTY OF ZIPPER}

A pocket $P K T\left(V_{i}, V_{j}\right)$ is said to be maximal with respect to $C\left(V_{0}, V_{q}\right)$ if $C\left(V_{i}, V_{j}\right)$ is not contained in another pocket $P K T\left(V_{k}, V_{m}\right)$, where $0 \leq i<j \leq q$, and $0 \leq k<m \leq q$. Let an ordered list $\left(Z_{0}, Z_{1}, Z_{2}, \ldots, Z_{j}\right)$ be the sequence of all vertices in $C\left(V_{0}, V_{q}\right)$ such that $\operatorname{PKT}\left(Z_{i}, Z_{i+1}\right), 0 \leq i<j$, is maximal with respect to $C\left(V_{0}, V_{q}\right)$. The sequence of line segments $\left(L\left(Z_{0}, Z_{1}\right)\right.$, $\left.L\left(Z_{1}, Z_{2}\right), \ldots, L\left(Z_{j-1}, Z_{j}\right)\right)$ is said to be a zipper $Z P R\left(V_{0}, V_{q}\right)$ as illustrated in Fig. 1.

In this section, we show that $Z P R\left(V_{0}, V_{q}\right)$ is concave and non-self-intersecting. Our first lemma forms the basis for showing this property. In its proof and in all subsequent discussions, we use the following notations.

$V_{q+1}=$ the most recently visited vertex in $P$.

$V_{q}=$ the previous (counter-clockwise) vertex of $V_{q+1}$ in $P$.

$Z_{j}=$ the vertex that is most recently added into $Z P R\left(V_{0}, V_{q}\right)$.

$Z_{j-1}=$ the previous vertex of $Z_{j}$ in $Z P R\left(V_{0}, V_{q}\right)$.

$V_{*}=$ the previous vertex of $Z_{j}$ in $P$.
Lemma 3.1. Let $Z P R\left(V_{0}, V_{q}\right)=\left(L\left(Z_{0}, Z_{1}\right), L Z_{1}\right.$, $\left.\left.Z_{2}\right), \ldots, L\left(Z_{j-1}, Z_{j}\right)\right)$ and $0<q<n$. Any vertex $V_{k}$ in the chain $C\left(Z_{r}, V_{q}\right)$ must be to the right of $L\left(Z_{i}, Z_{i+1}\right), 0$ $\leq i<r<j$, if $V_{k} \neq Z_{i+1}$.

Proof. The proof will be by the induction on the subscript $i$ of a zipper vertex $Z_{i}$ in $Z P R\left(V_{0}, V_{q}\right)$. Let $Z_{-1}$ be a point on the horizontal line containing $Z_{0}$ such that $Z_{-1}$ lies to the right of $Z_{0}$. Let $L_{i}$ be the line containing $L\left(Z_{i-1}, Z_{i}\right), i=0,1,2, \ldots, j . L_{i}$ partitions the plane into two half planes. Let $L H P_{i}$ be the half plane to the left of $L\left(Z_{i-1}, Z_{i}\right)$ and $R H P_{i}$ be the other.

$i=0$. Since $V_{0}$ is extreme, $V_{0}$ coincides with $Z_{0}$. By the way in which $V_{0}$ is chosen, the $Y$ coordinate of $V_{0}$ is not greater than the $Y$ coordinate of any other vertex in $P$. Therefore, $C\left(V_{0}, V_{q}\right)$ cannot pass through $L H P_{0}$. Now, $R H P_{0}$ is partitioned by $L_{1}$ into two regions, $R H P_{0} \cap L H P_{1}$ and $R H P_{0} \cap R H P_{1}$. We need to show that $C\left(Z_{1}, V_{q}\right)$ cannot be in $R H P_{0} \cap L H P_{1}$. Suppose that some vertices in $C\left(Z_{1}, V_{q}\right)$ are in $R H P_{0} \cap L H P_{1}$. Let $W$ be the vertex in $C\left(Z_{1}, V_{q}\right)$ such that $C\left(Z_{0}, W\right)$ is to the right of $L\left(Z_{0}, W\right)$. Clearly, $P K T\left(Z_{0}, W\right)$ contains $C\left(Z_{0}, Z_{1}\right)$, which contradicts the maximality of $\operatorname{PKT}\left(Z_{0}, Z_{1}\right)$. Suppose that the lemma is true for $i=m$ $-1<j-2$.

$i=m$. We need to show that $C\left(Z_{i+1}, V_{q}\right)$ cannot be in

$$
R=\left[\bigcap_{p=0}^{m} R H P_{p}\right] \cap L H P_{m+1}
$$

as shown in Fig. 2.

Suppose that some vertices in $C\left(Z_{m+1}, V_{q}\right)$ are in $R$. Let $W$ be the vertex in $C\left(Z_{m+1}, V_{q}\right)$ such that $C\left(Z_{m}, W\right)$ is to the right of $L\left(Z_{m}, W\right) . P K T\left(Z_{m}, W\right)$ contains $C\left(Z_{m}\right.$, $\left.Z_{m+1}\right)$, which contradicts the maximality of $P K T\left(Z_{m}\right.$, $\left.Z_{m+1}\right)$.

As illustrated in Fig. 3, the property described in Lemma 3.1 does not necessarily hold true unless $V_{0}$ is an extreme vertex of $P$. We next state the lemmas characterizing a $Z P R\left(V_{0}, V_{q}\right)$, the proofs of which are direct consequences of Lemma 3.1 .

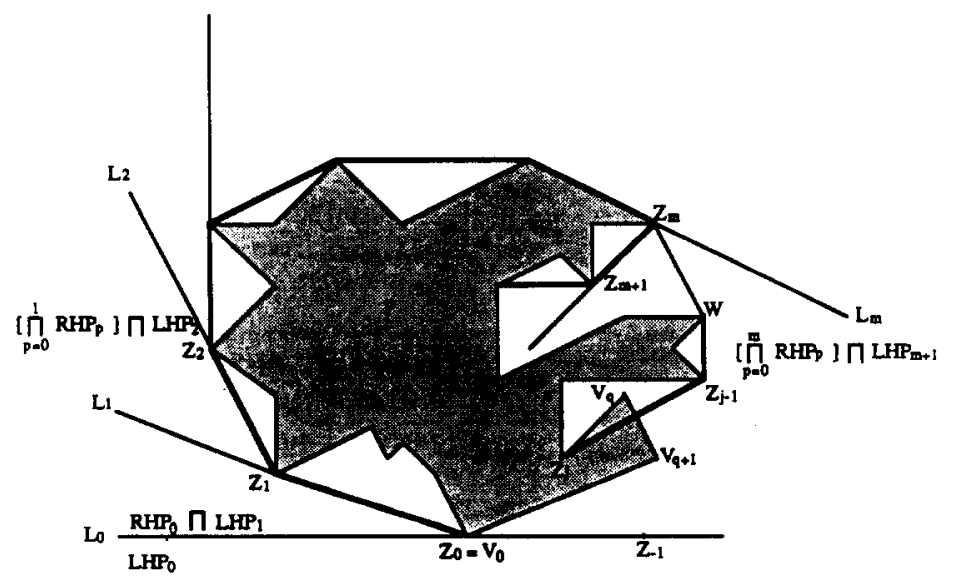

Fig. 2. $C\left(V_{m+1}, V_{q}\right)$ cannot be in $\left[\bigcap_{p=0}^{m} R H P_{p}\right] \cap L H P_{m+1}$. 


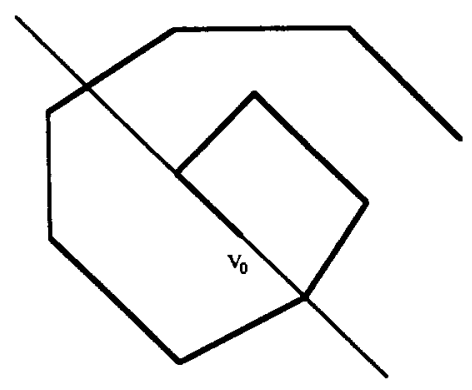

Fig. 3. Lemma 3.1 does not hold true if $v_{0}$ is not extreme.

Lemma 3.2. Let $Z P R\left(V_{0}, V_{q}\right)=\left(L\left(Z_{0}, Z_{1}\right), L\left(Z_{1}\right.\right.$, $\left.\left.Z_{2}\right), \ldots, L\left(Z_{j-1}, Z_{j}\right)\right)$. The internal angle ANGLE $\left(Z_{i}\right.$, $Z_{i+1}, Z_{i+2}$ ) between two consecutive line segments $L\left(Z_{i}, Z_{i+1}\right)$ and $L\left(Z_{i+1}, Z_{i+2}\right), 0 \leq i \leq j-2$, is strictly between 0 and 180 degrees.

Lemma 3.3. A $Z P R\left(V_{0}, V_{q}\right)$ is not self-intersecting.

Finally, we show that a zipper vertex $Z_{k}$ cannot be in a pocket $P K T\left(Z_{i}, Z_{i+1}\right)$ if $k \neq i$ and $k \neq i+1$. We use this property to update $Z P R\left(V_{0}, V_{q}\right)$.

Lemma 3.4. Let $Z P R\left(V_{0}, V_{q}\right)$ be $\left(L\left(Z_{0}, Z_{1}\right), L\left(Z_{1}\right.\right.$, $\left.\left.Z_{2}\right), \therefore, L\left(Z_{j-1}, Z_{j}\right)\right)$.

Then,

$$
Z_{k} \cap \operatorname{PKT}\left(Z_{i}, Z_{i+1}\right)=\begin{aligned}
& Z_{k} \text { if } k=i \text { or } i+1 \\
& \varnothing \text { otherwise }
\end{aligned}
$$

for all $0 \leq i<j$ and $0 \leq k \leq j$.

Proof. Suppose that $Z_{k} \cap P K T\left(Z_{i}, Z_{i+1}\right) \neq \varnothing$ for some $k \neq i$ and $k \neq i+1$. Then either $P$ is not simple or $V_{0}$ is not an extreme point. $\square$

\section{LPDATING OF ZIPPER}

Consider the relationship between two line segments $L\left(Z_{j-1}, Z_{j}\right)$ and $E\left(V_{*}, Z_{j}\right)$ As illustrated in Fig. 4, the vertex $V_{q+1}$ can be in any one of the four quadrants formed by the extensions of these two line segments. The quadrants are:

Q1a: to the right of $L\left(Z_{j-1}, Z_{j}\right)$ and to the right of $E\left(V_{*}, Z_{j}\right)$

Q1b: to the right of $L\left(Z_{j-1}, Z_{j}\right)$ and to the left of $E\left(V_{*}\right.$, $\left.Z_{j}\right)$

Q2a: to the left of $L\left(Z_{j-1}, Z_{j}\right)$ and to the right of $E\left(V_{*}\right.$, $\left.Z_{j}\right)$

Q2b: to the left of $L\left(Z_{j-1}, Z_{j}\right)$ and to the left of $E\left(V_{*}\right.$, $\left.Z_{j}\right)$

If $V_{q+1}$ is in Q1b, it is also in $P K T\left(Z_{j-1}, Z_{j}\right)$. By Lemma 2.1, $V_{q+1}$ and its clockwise vertices in $P K T\left(Z_{j-1} . Z_{j}\right)$ can be deleted. Otherwise, we need to show if the existing zipper vertices are to be deleted or kept to advance to $V_{q+1}$. The following three lemmas as illustrated in Fig. 5 are useful for the updating of $\operatorname{ZPR}\left(V_{0}, V_{q}\right)$.

Lemma 4.1. Let $Z P R\left(V_{0}, V_{q}\right)=\left(L\left(Z_{0}, Z_{1}\right), L\left(Z_{1}\right.\right.$, $\left.\left.Z_{2}\right), \ldots, L\left(Z_{j-1}, Z_{j}\right)\right)$ and $V_{q}=Z_{j} \neq V_{0}$. All pockets $P K T\left(Z_{i}, Z_{i+1}\right), 0 \leq i<j$, are maximal with respect to $C\left(V_{0}, V_{q+1}\right)$, if $V_{q+1}$ is in Qla.

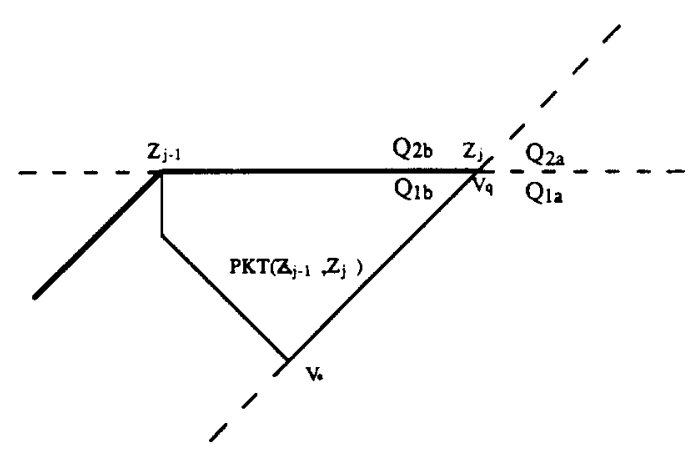

Fig. 4. Possible locations of vertex $V_{q+1}$

Proof. If $V_{q+1}=V_{0}$ then $Z P R\left(V_{0}, V_{q}\right)$ together with $E\left(V_{q}, V_{q+1}\right)$ forms a convex polygon since $V_{q+1}=V_{0}$ and $Z P R\left(V_{0}, V_{q}\right)$ is concave and non-selfintersecting. Therefore, the result follows immediately.

Let us consider the case for $V_{q+1} \neq V_{0}$. Since $Z P R\left(V_{0}, V_{q}\right)$ implies that $P K T\left(Z_{i}, Z_{i+1}\right) .0 \leq i<j$, is maximal with respect to $C\left(V_{0} . V_{q}\right)$, all we need to show is that $E\left(Z_{j}, V_{q+1}\right)$ is $\operatorname{PKT}\left(Z_{j}, V_{q+1}\right)$ and is maximal with respect to $C\left(V_{0}, V_{q+1}\right)$. First we show $E\left(Z_{j}, V_{q+1}\right)$ $\cap P K T\left(Z_{i}, Z_{i+1}\right) \neq E\left(Z_{j}, V_{q+1}\right)$ for any $0 \leq i<j$. By Definition 2.5, $V_{q+1}$ cannot be in $P K T\left(Z_{j-1}, Z_{j}\right)$ since $V_{q+1}$ is in Q1a. From Lemma $3.4, Z_{j}$ cannot be in $P K T\left(Z_{i}, Z_{i+1}\right)$ for any $0 \leq i<j-1$. Therefore, $E\left(Z_{j}\right.$, $\left.V_{q+1}\right) \cap P K T\left(Z_{i}, Z_{i+1}\right) \neq E\left(Z_{j}, V_{q+1}\right)$ for any $0 \leq i<$

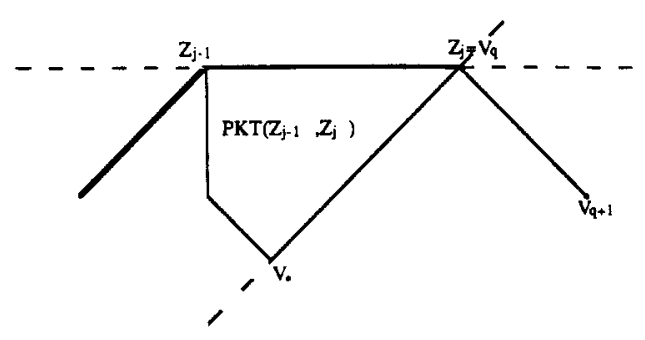

(a)

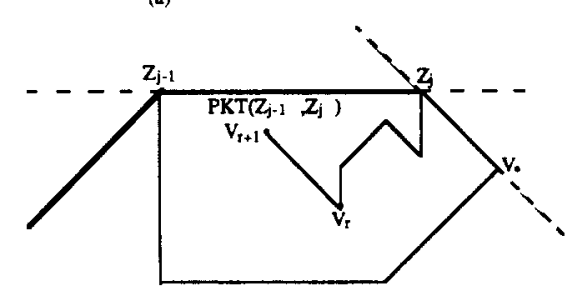

(b)

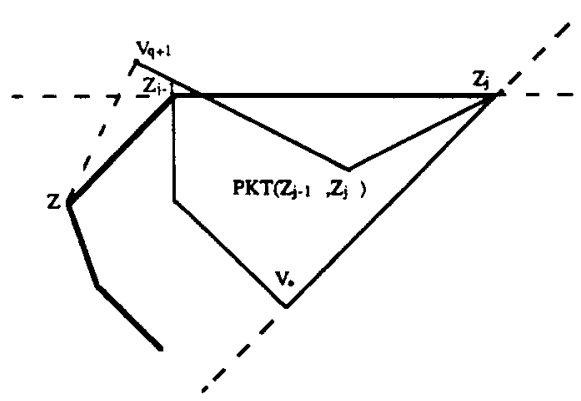

Fig. 5. Updating zipper vertices. (a) Illustration of Lemma 4.1. (b) Illustration of Lemma 4.2. (c) Illustration of Lemma 4.3. 
$j$. Finally, there does not exist a vertex $V_{r}$ in $C\left(V_{0}, V_{*}\right)$ such that $C\left(V_{r}, V_{q+1}\right)$ and $L\left(V_{r}, V_{q+1}\right)$ form a pocket $P K T\left(V_{r}, V_{q+1}\right)$ since $Z P R\left(V_{0}, V_{q}\right)$ is concave and $P$ is simple. Hence, the result follows. $\square$

Lemma 4.2. Let $Z P R\left(V_{0}, V_{q}\right)=\left(L\left(Z_{0}, Z_{1}\right), L\left(Z_{1}\right.\right.$, $\left.\left.Z_{2}\right), \ldots, L\left(Z_{j-1}, Z_{j}\right)\right)$. If $C\left(V_{q}, V_{r}\right), r>q$, is in $\operatorname{PKT}\left(Z_{j-1}, Z_{j}\right)$, then $V_{r+1}$ is also in $\operatorname{PKT}\left(Z_{j-1}, Z_{j}\right)$ unless $V_{r+1}$ is to the left of $L\left(Z_{j-1}, Z_{j}\right)$.

Proof. Since $P$ is simple, $C\left(V_{q}, V_{r+1}\right)$ can get out of $\operatorname{PKT}\left(Z_{j-1}, Z_{j}\right)$ only through $L\left(Z_{j-1}, Z_{j}\right)$.

Lemma 4.3. Let $Z P R\left(V_{0}, V_{q}\right)=\left(L\left(Z_{0}, Z_{1}\right), L\left(Z_{1}\right.\right.$, $\left.\left.Z_{2}\right), \ldots, L\left(Z_{j-1}, Z_{j}\right)\right)$. Then $\operatorname{PKT}\left(Z_{j-1}, Z_{j}\right)$ is not maximal with respect to $C\left(V_{0}, V_{q+1}\right)$, if $V_{q+1}$ is in quadrant Q2a or Q2b.

Proof. $A N G L E\left(Z_{j-1}, Z_{j}, V_{q+1}\right)$ is greater than or equal to 180 degrees since $V_{q+1}$ is in Q2a or Q2b. Since $Z P R\left(V_{0}, V_{q}\right)$ is concave and non-selfintersecting, there must exist a vertex $Z$ in $Z P R\left(V_{0}, V_{q}\right)$ such that $L(Z$, $\left.V_{q+1}\right)$ and $C\left(Z, V_{q+1}\right)$ form a pocket $P K T\left(Z, V_{q+1}\right)$. Clearly, $P K T\left(Z, V_{q+1}\right)$ contains $C\left(Z_{j-1}, Z_{j}\right) . \square$

\section{THE ALGORITHM AND ITS ANALYSIS}

Our linear algorithm for finding the convex hull of a simple polygon $P$ takes $V_{i}, i=0,1, \ldots, n-1$, as input and constructs a $Z P R\left(V_{0}, V_{q}\right)$ with vertices $Z_{j}$.

\section{Algorithm 5.1}

Step 0. $\quad Z_{0} \leftarrow V_{0}, Z_{1} \leftarrow V_{1}, j \leftarrow 1, q \leftarrow 1$. while $\left(V_{q} \neq V_{\mathrm{o}}\right)$ do;

Step 1, if $V_{q+1}$ is to the right of $L\left(Z_{j-1}, Z_{j}\right)$, then do:

Step la. if $V_{q+1}$ is to the right of $E(V, Z)$ then $j \leftarrow j+1, Z_{j} \leftarrow V_{4+1}, q \leftarrow q+1$.

Step Ib. else while $\left(V_{4+1}\right.$ is on or to the right of $\left.L\left(Z_{j-1}, Z_{j}\right)\right)$ do;

$q \leftarrow q+1$

end

end

Step 2 else do;

while $\left(Z_{j} \neq V_{0}\right.$ and $Z_{j-1}$ is not to the right of $\left.L\left(Z_{j}, V_{q+1}\right)\right) \mathrm{do}$;

$j \leftarrow j-1$

end.

$j \leftarrow j+1, Z_{j} \leftarrow V_{q+1}, q \leftarrow q+1$.

end

end

Step 3. Stop.

We show the correctness of Algorithm 5.1 with the following lemma.

Lemma 5.1. Algorithm 5.1 constructs $Z P R\left(V_{0}, V_{q}\right)$ correctly.

Proof. The proof will be by induction on the number of times Step 1 is reached. Initially, the statement is trivially satisfied by Step 0 of the algorithm. Suppose that the lemma is true when Step 1 is executed $m$ times. Then, there are three cases:

(1) Case 1a: $V_{q+1}$ is in Q1a

(2) Case $1 \mathrm{~b}: V_{q+1}$ is in Q1b

(3) Case 2: $V_{q+1}$ is in Q2a or Q2b.
Case 1a: $V_{4+1}$ qualifies as a zipper vertex if $P K T\left(Z_{j}\right.$, $\left.V_{4+1}\right)$ is maximal with respect to $C\left(V_{0,}, V_{4+1}\right)$. Since $V_{4+1}$ is in quadrant Q1a, by Lemma 4.1, $\operatorname{PK} T\left(Z_{j}, V_{4+1}\right)$ is maximal. Indeed, Step la takes $V_{q+1}$ as the new $Z_{i}$. Since the correct vertex is added to the zipper the next time Step 1 is reached, the induction holds. Now, Lemma 4.1 requires the precondition that $V_{4}$ equals $Z_{j}$. This precondition is satisfied iteratively after executing Step 1a or Step 2. After executing Step $1 \mathrm{~b}$, though $V_{q} \neq Z_{j}$, the control must go to Step 2 because $V_{q+1}$ cannot be to the right of $L\left(Z_{j-1}, Z_{j}\right)$. Hence, the precondition for Lemma 4.1 is always satisfied.

Case 1b: Because $V_{q+1}$ is in quadrant $Q 1 \mathrm{~b}$, by Definition 2.5, $V_{4+1}$ is in $\operatorname{PKT}\left(Z_{j-1}, Z_{j}\right)$. Therefore, $V_{4+1}$ should not be a zipper vertex. Furthermore, by Lemma 4.2 , all the subsequent vertices in $\operatorname{PKT}\left(Z_{j-1}, Z_{j}\right)$ should not be in the zipper $Z P R\left(V_{0}, V_{q}\right)$ either. This is precisely what Step $1 b$ does. Since no zipper vertex is added, the next time Step 1 is reached, $Z P R\left(V_{0}, V_{4}\right)$ is still correct.

Case 2: Step 2 deletes $Z_{j}$ since $\operatorname{PKT}\left(Z_{j-1}, Z_{j}\right)$ is not maximal with respect to $C\left(V_{0}, V_{4+1}\right)$ by Lemma 4.3. The old $Z_{j-1}$ becomes the new $Z_{j}$. This process is repeated until either $Z_{j}=$ $Z_{0}$ or $Z_{j-1}$ is to the right of $L\left(Z_{p}, V_{q+1}\right)$. At that point $P K T\left(Z_{j}, V_{q+1}\right)$ is maximal with respect to $C\left(V_{0}, V_{4+1}\right)$, because $Z P R\left(V_{0}\right.$, $\left.V_{4+1}\right)$ is concave and non-self-intersecting. Hence, the lemma is true.

When $V_{4}$ coincides with $V_{0}$, Step 3 terminates the $q$ algorithm, and the lemma is still true by the induction hypothesis.

Since $Z P R\left(V_{0}, V_{q}\right)$ is concave and non-selfintersecting, it must form a convex polygon $P_{r}$ containing $P$ if $V_{q}=V_{0}$. Since every vertex of $P_{c}$ is a vertex of $P$, it is clear that $P_{c}$ is the smallest convex polygon containing $P$. By Definition 2.4, $P_{c}$ must be the convex hull of a simple polygon $P$.

Theorem 5.1. Algorithm 5.1 finds the convex hull of a simple polygon $P$ with $n$ vertices in $O(n)$ time.

Proof. The algorithm moves forward, except in Step 2 , until $V_{0}$ is revisited. Step 2 is executed at most a total of $n-3$ times.

\section{CONCLUDING REMARKS}

Algorithm 5.1 removes the vertices that cause selfintersection $^{(8)}$ in $\mathrm{CH}$-POL. ${ }^{(9)}$ It is shorter than the version by Graham and $\mathrm{Yao}^{(6)}$ when both the Left Hull and the Right Hull are taken into account.

\section{SUMMARY}

A new linear algorithm for finding the convex hull of a simple polygon is given. Based on the original idea by 
Sklansky, ${ }^{(7)}$ our version is easy to understand. Adopting the form of CH-POL by Toussaint and Avis, ${ }^{(9)}$ the presentation is concise. As shown in the Appendix, a PASCAL implementation of the algorithm itself is only half a page long.

In the paper, we define a "zipper" as a non-selfintersecting, concave chain. Choosing an extreme vertex of the polygon as the initial zipper, we update it by classifying a vertex of the given polygon by one of three cases. Case 1: vertex of the given polygon is added to the zipper. Case 2: vertex of the given polygon is not added to the zipper. Case 3: zipper vertex is deleted. We show that, after a complete traversal of the given polygon, the zipper thus constructed is the convex hull.

Acknowledgement-The authors wish to thank J. D. Wolter and $H$. C. Lee for their critical reading of the manuscript and their constructive suggestions. J. D. Wolter implemented Algorithm 5.1 in several languages. His version in PASCAL is supplied in the Appendix.

\section{REFERENCES}

1. S. Ghosh and R. Shyamasundar. A linear time algorithm for obtaining the convex hull of a simple polygon. Pattern Recognition 16, 587-592 (1983).

2. R. Shyamasunder, Note on a linear time algorithm for obtaining the convex hull of a simple polygon, private communication. 28 August (1984).

3. T. Woo and S. Shin. Counterexamples, private communications, 10 July and 15 October (1984).

4. D. McCallum and D. Avis. A linear time algorithm for finding the convex hull of a simple polygon, Infor. Proc. Lett. 9, 201-205 (1979).

5. D. Lee, On finding the convex hull of a simple polygon. Int. J. Comput. Infor. Sci. 12, 2, 87-98 (1983).

6. R. Graham and F. Yao, Finding the convex hull of a simple polygon, J. Algorithms 4, 324-33! (1983).

7. J. Sklansky, Measuring concavity on a rectangular mosaic, IEEE Trans. Comput. 21, 1355-1364 (1972).

8. A. Bykat, Convex hull of a finite set of points in two dimensions, Infor. Proc. Lett. 7, 6. 296-298 (1978).

9. G. Toussaint and D. Avis, On a convex hull algorithm and its application to triangulation problems, Pattern Recognition 15, 23-29 (1982).

10. M. Orlowsky, On the condition for success of Sklansky's convex hull algorithm. Pattern Recognition 16, 579-586 (1983).

11. J. Sklansky, Finding the convex hull of a simple polygon, Pattern Recognition Lett. 1, 79-83 (1982).

12. G. Toussaint and $H$. El Gindy, A counterexample to an algorithm for computing monotone hulls of simple polygons, Pattern Recognition Lett. 1, 219-222 (1983).

\begin{abstract}
About the Author-Sung Y. SHIn is a Ph.D. candidate in Industrial and Operations Engineering at the University of Michigan. His research interests include computational geometry, algorithm design and analysis, CAD/CAM, and information systems.

After receiving his B.S. degree in 1970 from Hanyang University in Seoul, Korea. Mr. Shin was involved in developing computer-integrated manufacturing systems for various industries in Korea.

About the Author-Tony C. Woo received his B.S., M.S. and Ph.D. degrees. in Electrical Engineering. from the University of Illinois in 1968, 1974 and 1975, respectively.

Joining the University of Michigan in 1977, Dr. Woo is currently Associate Professor in Industrial and Operations Engineering. He teaches courses in computer graphics, and geometric modeling. His research is in the design of geometric algorithms for CAD, CAM and robotics applications. He is the 1985 recipient of the TRW Foundation Award in Manufacturing Engineering.
\end{abstract}

\title{
APPENDIX
}

program main (input, output);

var $X, Y$ : array $[0 . .50]$ of real; $\{$ coordinates of points:

$V, Z$ : array $[0 . .50]$ of integer; \{polygon and hull\}

$q, j:$ integer; $\quad$ index into polygon and hull

$n$ : integer; number of vertices:

$i$ integer; \{loop index;

\{Is point $\mathrm{p}$ to the left of Line $(a, b)$ ?\}

function left $(p, a, b$ : integer) :boolean;

begin

end;

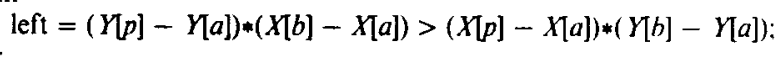

\{Is point $\mathrm{p}$ to the right of Line $(a, b)$ ?\}

function right $(p, a, b$ : integer $)$ boolean;

begin

end:

right $=(Y[p]-Y[a]) *(X[b]-X[a])<(X[p]-X[a]) *(Y[h]-Y[a])$

\{Read in the Polygon\}

procedure readin;

var $i: \quad$ integer;

$W: \quad$ array $[0 . .50]$ of integer;

$m x, m y:$ real;

$m i$ integer; 


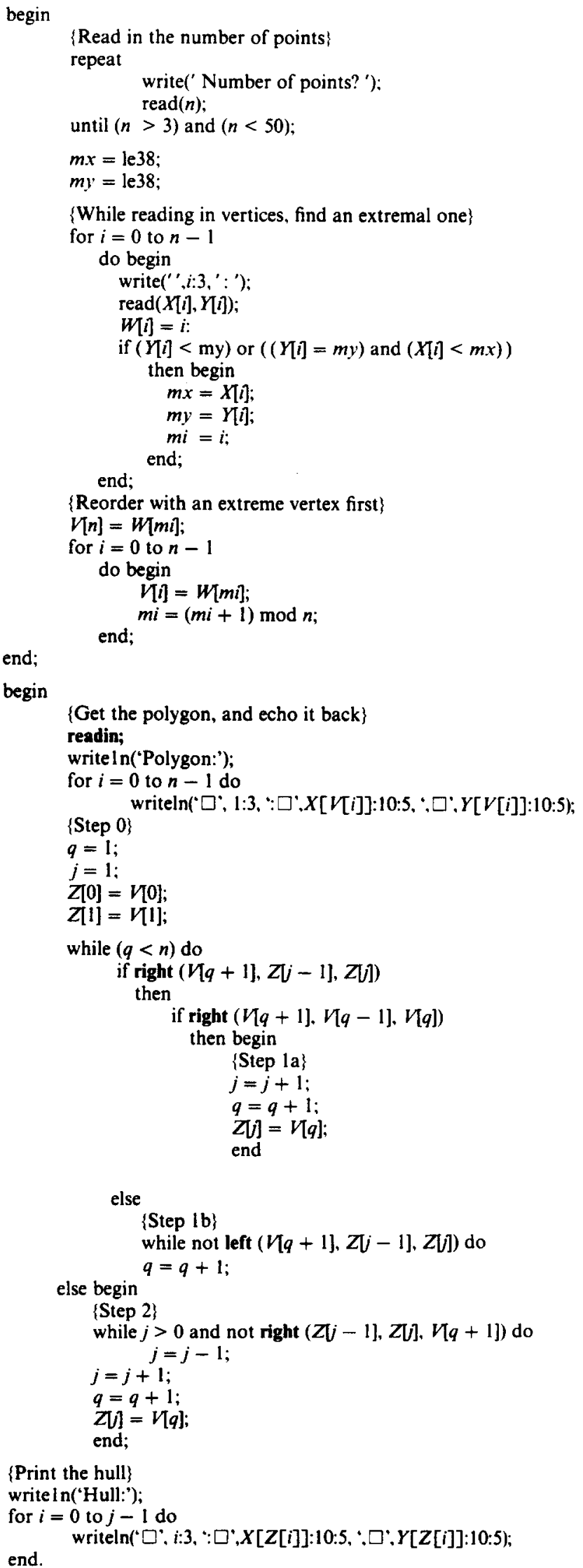

